\title{
La revue scientifique : un élément d'analyse des sciences humaines et sociales
}

\author{
Etienne Gérard \\ Sociologue, IRD, 8 rue voltaire \\ 93100 Montreuil, France \\ Etienne.gerard@ird.fr \\ Mina Kleiche-Dray \\ Historienne des sciences, IRD \\ IRD, Cicéron 609, col. Los Morales, Pola,co \\ CP 11530 Mexico DF, Mexique \\ minakleiche@yahoo.fr
}

\section{Résumé}

L'article se propose d'aborder la question de la structuration du champ des sciences humaines et sociales, en prenant pour "entrée" les revues scientifiques de ces disciplines, en particulier dans le cas de la production scientifique marocaine. Il s'agit, à travers l'analyse de la structure et du fonctionnement d'un ensemble de revues de sciences humaines et sociales, de montrer que cette production constitue un indice de la structuration de ce champ scientifique. L'article montre notamment qu'à une focalisation disciplinaire correspond un certain recentrage des revues sur elles-mêmes - donc un certain regroupement des chercheurs autour d'objets scientifiques, de normes de publication, ou encore de modèles de traitement de ces objets. Par ailleurs, des supports de publication comme les revues se resserrent autour de certains objets scientifiques, et doivent probablement l'importance croissante de leur noyau de contributeurs à cette spécialisation et à la visibilité peu à peu acquise par la revue; en somme, à un certain monopole de la production scientifique.

Mots clefs: Maroc - Sciences humaines et sociales - Revues scientifiques - Champ - Structure

\section{Introduction}

Au Maroc, comme dans la majorité des pays occidentaux, la revue scientifique est le principal espace de publication retenu par les chercheurs pour faire connaître leurs résultats de recherches, le principal organe aussi de diffusion de ces résultats auprès de la communauté des chercheurs. Selon une recherche que nous avons 
réalisée au Maroc sur la production scientifique globale (de 1995 à 2001), plus de $50 \%$ des travaux scientifiques publiés prennent en effet place dans ce support (graphique 1, page suivante).

La revue scientifique offre donc un large panorama, même s'il n'est pas exhaustif, des domaines de recherches explorés et des sujets abordés par les chercheurs des différentes disciplines. L'ensemble des revues présente par ailleurs une image de la production scientifique en termes quantitatifs : il renseigne sur l'état de cette production du point de vue de la diversité et de la fréquence des sujets traités, et sur la communauté de chercheurs mobilisés pour aborder ces sujets. La fréquence de parution d'une revue instruit ainsi non seulement de l'intérêt accordé, au fil des années, à un domaine ou un thème donné de recherche, mais aussi des ressources mobilisées (en nombre de chercheurs) pour cette exploration. À titre d'exemple, après avoir été objets d'investigations constantes durant de nombreuses années, les recherches relatives à l'éducation paraissaient, il y a quelques années, de plus en plus rares ; et, lorsqu'elles se poursuivaient, elles ne suffisaient plus à alimenter la revue spécialisée sur ces questions, Attadriss, qui ne paraissait plus que très rarement (par exemple, sept ans séparaient le numéro de 1999 du précédent).

Une étude sur les principales revues en sciences humaines et sociales peut donc apporter des informations, non seulement sur les recherches conduites et sur leur publication, mais aussi sur l'état de la production, en termes de volume et de structuration. Par hypothèse, une telle étude nous semblait pouvoir offrir des réponses à la double question de savoir ce qui se publie et comment les recherches sont publiées, notamment à quelle fréquence et par quels chercheurs (identifiés selon leur discipline, leur institution d'appartenance, marocaine ou étrangère). De cette hypothèse a découlé une autre question : une analyse des revues scientifiques peut-elle renseigner sur les caractéristiques du champ des sciences humaines et sociales au Maroc, en particulier sur la structuration de ce milieu scientifique?

In fine, c'est cette question qui a motivé les recherches dont nous exposons ici des résultats ${ }^{1}$.

L'approche et la méthode ont été doubles : d'une part dépouiller des revues scientifiques et en analyser le contenu, d'autre part aborder les revues comme "contenants" - et non plus seulement comme véhicules de contenus scientifiques - ayant une structure particulière, dont l'analyse devrait permettre de comprendre le fonctionnement et, au-delà, des logiques structurantes du champ scientifique.

1 Nous avons développé une partie de la recherche, en particulier sur la nature disciplinaire de la production scientifique, dans un autre article (Gérard \& Kleiche-Dray, 2006). Nous nous consacrerons ici exclusivement aux revues scientifiques. 
Il s'agissait donc de "traiter" ce matériau scientifique non plus comme un réceptacle ou comme une collection de produits scientifiques individuels, mais de l'interroger pour tenter de déceler si les revues scientifiques ne sont pas le produit de "groupes" de chercheurs - donc de relations entre eux —, davantage que la simple agrégation d'articles publiés in nomine par des chercheurs, sinon isolés, du moins sans "relations de production scientifique" entre eux.

\section{Graphique 1 \\ Volume et part de chaque type de production scientifique dans la base recensée (1995-2001)}

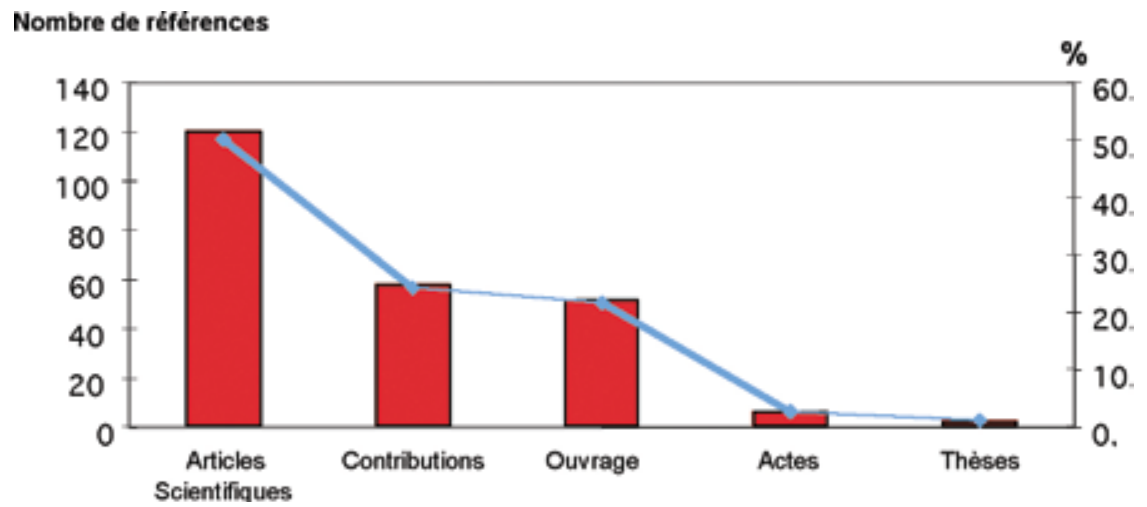

Pour tenter d'apporter des réponses à ces questions et avancer dans la validation de ces hypothèses, nous avons principalement retenu sept revues marocaines (après une première exploration de quatorze), en fonction de trois critères principaux : leur parution de 1995 à 2001 (certaines, comme la Revue MarocEurope, Attadriss ou la Revue de géographie du Maroc ont finalement été écartées compte tenu du faible nombre de publications durant cette période), leur représentativité disciplinaire, appréciée sur la base de l'ensemble des productions pour chaque discipline et sur la base d'entretiens avec des chercheurs, enfin 1'“ancrage institutionnel" des revues (encart 1). Pour chaque numéro des revues sélectionnées, le dépouillement a consisté à dresser un fichier dans lequel devaient figurer, outre les coordonnées du numéro : le titre de l'article — auquel ont été affectés des mots-clefs thématiques - l'auteur correspondant et sa discipline. Le dépouillement concerne les revues et leurs numéros suivants. 


\section{Encart 1}

Les revues de Sciences humaines et sociales étudiées (I995-200i)

\begin{tabular}{|c|c|}
\hline REMALD & \\
\hline $\begin{array}{l}\text { Période : } 1995 \text { à } 2001 \\
\text { Nombre de numéros : } 27 \text { (n } 10 \text { à n³8-39) } \\
\text { Nombre d'articles : } 349 \\
\text { Nombre de contributeurs : } 97\end{array}$ & $\begin{array}{c}\text { REVUE JURIDIQUE POLITIQUE ET } \\
\text { ECONOMIQUE DU MAROC }\end{array}$ \\
\hline PROLOGUES & $\begin{array}{l}\text { Période : } 1995 \text { à } 2000 \\
\text { Nombre de numéros : } 4 \text { (28 à 31-32) } \\
\text { Nombre d'articles : } 48 \\
\text { Nombre de contributeurs : } 42\end{array}$ \\
\hline $\begin{array}{l}\text { Période : } 1995 \text { à } 2001 \\
\text { Nombre de numéros : } 16\left(\mathrm{n}^{\circ} 4 \text { à n } 21+\text { un n }^{\circ}\right. \\
\text { H.S) } \\
\text { Nombre d'articles : } 239 \\
\text { Nombre de contributeurs : } 122\end{array}$ & $\begin{array}{l}\text { CAHIERS DE LA FONDATION } \\
\text { ABDERRAHIM BOUABID }\end{array}$ \\
\hline ANNALES MAROCAINES D'ECONOMIE & $\begin{array}{l}\text { Période : janvier } 97-\text { mars } 99 \\
\text { Nombre de numéros : } 20 \text { (1 à 27) } \\
\text { Nombre d'articles : } 98 \\
\text { Nombre de contributeurs : } 68\end{array}$ \\
\hline $\begin{array}{l}\text { Période : } 1995 \text { à } 1998 \\
\text { Nombre de numéros : } 11 \text { (n } 11 \text { à n’22-23) } \\
\text { Nombre d'articles : } 126 \\
\text { Nombre de contributeurs : } 93 \\
\text { HESPERIS TAMUDA }\end{array}$ & $\begin{array}{l}\text { Période : } 1996 \text { à } 2001 \\
\text { Nombre de numéros : } 9 \text { (n¹-2 à n¹3) } \\
\text { Nombres d'articles : } 41 \\
\text { Nombre de contributeurs : } 33\end{array}$ \\
\hline $\begin{array}{l}\text { Période : } 1996 \text { à } 1997 \\
\text { Nombre de numéros : } 4 \text { (n`34 à n³7) } \\
\text { Nombre d'articles : } 51 \\
\text { Nombre de contributeurs : } 49\end{array}$ & \\
\hline
\end{tabular}

Les spécificités disciplinaires des revues apparaissent comme suit, après relevé de tous les articles parus durant la période : 


\section{Tableau 1. Spécificités disciplinaires dominantes des revues dépouillées}

\begin{tabular}{l|l}
\hline Revue & Dominantes disciplinaires \\
\hline Remald & Droit, économie, sciences politiques \\
\hline Prologues & Sociologie, sciences politiques, littérature \\
\hline Annales marocaines d'économie & Économie, socio-économie \\
\hline Hesperis Tamuda & Histoire \\
\hline Revue juridique politique et économique du Maroc & Droit, économie, sociologie \\
\hline $\begin{array}{l}\text { Cabiers de la fondation A. Bouabid } \\
\text { Études maghrébines }\end{array}$ & $\begin{array}{l}\text { Sciences politiques, économie, sociologie } \\
\text { Histoire, philosophie, anthropologie }\end{array}$ \\
\hline
\end{tabular}

Parmi ces revues peuvent être distinguées celles qui sont à forte dominante disciplinaire (comme Remald pour les questions de droit) ou plus "ouvertes" de ce point de vue (comme Prologues, Études maghrébines ou la Revue juridique, politique et économique marocaine (RJPEM), qui accueillent des articles de différentes disciplines : sociologie, sciences politiques, anthropologie, histoire, etc.). Les graphiques suivants en donnent une illustration pour deux revues incluses dans un champ disciplinaire similaire, Remald et RJPEM.

\section{Graphiques 2 et 3}

\section{Répartition des articles selon la discipline des auteurs dans les revues Remald et RJPEM}

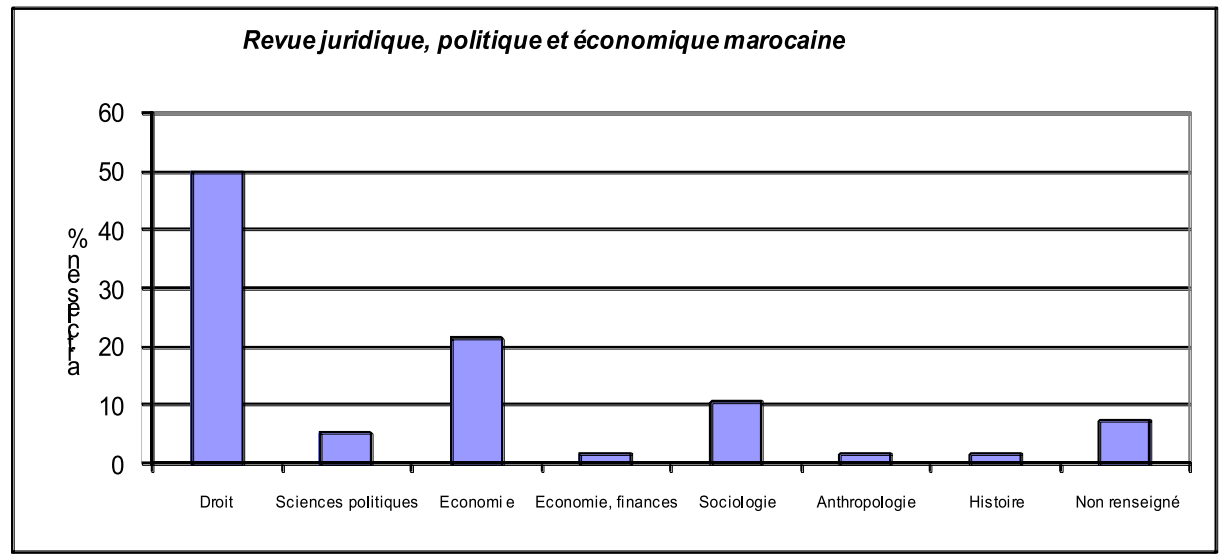




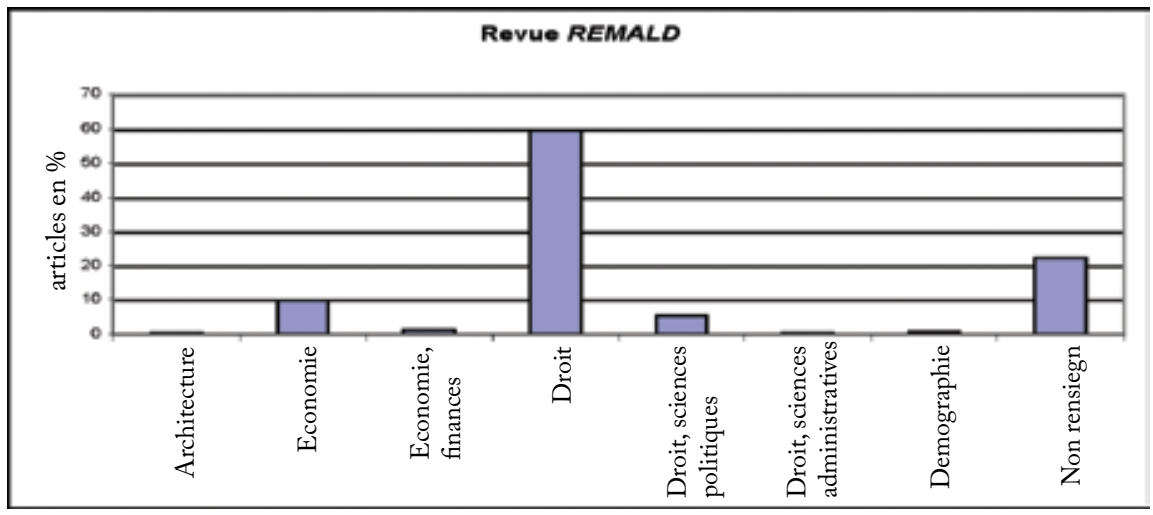

Les revues se différencient aussi selon que la publication est assurée par leur université d'appartenance (comme les revues des Facultés de lettres, sciences humaines et sociales de Fès, Oujda ou Marrakech — pour ne citer que celles-là) ou par une institution non universitaire, moins directement liée à l'enseignement supérieur (par exemple Prologues ou Les Cabiers de la Fondation A. Bouabid).

La plupart des revues ici retenues (Remald, Prologues, les Annales marocaines d'économie, Hesperis tamuda, la Revue juridique politique et économique du Maroc, les Cabiers de la fondation A. Bouabid et les Études maghrébines) ont par ailleurs une parution irrégulière. Certaines sont plutôt annuelles (la plupart), d'autres plutôt mensuelles (les Cabiers de la fondation A. Bouabid), d'autres encore comptent quelques numéros par an (Remald).

\section{La Revue Scientifique : Une Structure de Publication en "noyau" et "cercles" de Contributeurs}

L'une des questions principales posées pour appréhender la structuration de la production scientifique a été de savoir comment "fonctionne" chaque revue : repose-t-elle sur une quelconque "communauté de chercheurs" ? Si, oui, celle-ci est-elle homogène ou hétérogène d'un numéro à l'autre et dans le temps ? Plus encore : peut-on, en termes de structuration des revues, identifier une règle ou un schéma de fonctionnement qui s'applique à toutes, quel que soit leur champ disciplinaire respectif? La réponse n'est, a priori, pas donnée. Rien ne permet, en effet, de préjuger que toutes les revues publient selon les mêmes règles, qu'elles comportent les mêmes structures, ou encore qu'elles soient alimentées en articles de manière identique.

Des indicateurs simples vont dans ce sens : toutes les revues nont pas de comité de lecture; toutes nont pas non plus de "focalisation disciplinaire". 
Deux indicateurs, en revanche, ne sont pas visibles sans analyse : le fait que des revues reposent, ou non, sur un nombre donné de contributeurs - une hypothèse conjointe voulant que ces derniers soient mutuellement identifiés et, parallèlement, le mode de publication des chercheurs — soit de manière privilégiée dans un nombre restreint de revues, soit, à l'inverse, dans le plus large éventail possible. Ces deux indicateurs se rejoignent bien sûr pour mettre au jour la configuration de la production scientifique — ses modes, ses règles, sa capacité à structurer le champ de recherches, ou encore le pouvoir de représentation que peuvent en tirer des chercheurs ou groupes de chercheurs.

Pour apprécier le fonctionnement des revues, nous avons, dans un premier temps, regardé si les articles sont toujours produits par des auteurs différents ou si, à l'inverse, ils sont principalement écrits par quelques contributeurs.

Nous avons donc relevé la production moyenne par auteur, sur l'ensemble des numéros dépouillés pour chaque revue. Notons en premier lieu qu'un simple comptage fait état d'un mode hétérogène de production d'une revue à l'autre. Par exemple, et pour prendre les cas extrêmes, les auteurs de la revue Hespéris Tamuda, répertoriés à travers 51 articles publiés en 1996-97, en avaient écrit, en moyenne, un chacun ; ceux de la revue Remald, examinée de 1995 à 2001 à travers ses 27 numéros, en avaient écrit, toujours en moyenne, près de trois (tableau 2).

\section{Tableau 2. Production moyenne d'articles par auteur dans les revues dépouillées}

\begin{tabular}{l|c|c|c|}
\multicolumn{1}{c|}{ Revue } & $\begin{array}{c}\text { Nombre } \\
\text { d'articles }\end{array}$ & $\begin{array}{c}\text { Nombre de } \\
\text { contributeurs }\end{array}$ & $\begin{array}{c}\text { Nombre } \\
\text { moyen articles/ } \\
\text { contributeurs }\end{array}$ \\
\hline Remald & 254 & 97 & 2,6 \\
\hline Prologues & 239 & 122 & 1,9 \\
\hline Annales marocaines d'économie & 126 & 93 & 1,3 \\
\hline Hesperis Tamuda & 51 & 49 & 1 \\
\hline Revue JPEM & 46 & 42 & 1,1 \\
\hline Cahiers de la fondation A. Bouabid & 98 & 68 & 1,4 \\
\hline Études maghrébines & 41 & 33 & 1,2 \\
Total & $\mathbf{8 5 5}$ & $\mathbf{5 0 4}$ & $\mathbf{1 , 7}$ \\
\hline
\end{tabular}

Ce simple constat permettait d'émettre l'hypothèse que chaque revue comptait des auteurs qui apportaient régulièrement leur contribution, d'autres plus épisodiquement, d'autres encore, de façon occasionnelle. Autrement dit, chaque revue comporterait ce que l'on désignera ici par un "noyau" de chercheurs 
rédigeant régulièrement des articles pour la revue — si ce n'est dans chaque numéro - , et des "cercles" de contributeurs plus occasionnels - des cercles que l'on pourrait sans doute aussi différencier selon la participation respective des auteurs à l'ensemble des publications de la revue. Pour le vérifier, on a procédé au comptage systématique des articles écrits par chaque auteur, sur l'ensemble des numéros de chaque revue. Un nombre modal d'articles par auteur a ainsi pu être identifié pour chaque revue. Enfin, des “classes" — ou nombre d'articles par auteur - ont été établies, permettant de figurer le volume de production pour les auteurs, la dispersion de ces derniers selon leur production et, également, la répartition des articles en fonction de ce volume respectif de production.

Pour les revues que nous avons dépouillées, ces caractéristiques se présentent de la façon suivante :

\section{Tableau 3. Nombre modal d'articles par auteur dans les revues dépouillées}

\begin{tabular}{|c|c|c|c|c|}
\hline Revue & $\begin{array}{c}\text { Nombre } \\
\text { minimum } \\
\text { d'articles/ } \\
\text { auteur }\end{array}$ & $\begin{array}{l}\text { Nombre } \\
\text { maximum } \\
\text { d'articles/ } \\
\text { auteur }\end{array}$ & $\begin{array}{l}\text { Nombre } \\
\text { modal } \\
\text { d'articles/ } \\
\text { auteur }\end{array}$ & “Classes” d'articles (1) \\
\hline Remald & 1 & 43 & 4 & $\begin{array}{c}{[1-3],[4-6],[7-9]} \\
{[10-12],[13 \text { et }+[}\end{array}$ \\
\hline Prologues & 1 & 20 & 2 & {$[1-2],[3-4],[5-8],[9$ et +[} \\
\hline $\begin{array}{l}\text { Annales } \\
\text { marocaines } \\
\text { d'économie } \\
\end{array}$ & 1 & 6 & 1 & {$[1],[2-3],[4$ et +[} \\
\hline Hesperis Tamuda & 1 & 2 & 1 & {$[1],[2]$} \\
\hline RJPEM & 1 & 3 & 1 & {$[1],[2],[3]$} \\
\hline $\begin{array}{l}\text { Cabiers de la } \\
\text { fondation } A \text {. } \\
\text { Bouabid }\end{array}$ & 1 & 11 & & {$[1-2],[3-4],[9$ et +[} \\
\hline $\begin{array}{l}\text { Études } \\
\text { maghrébines }\end{array}$ & 1 & 3 & 1 & {$[1],[2-3]$} \\
\hline
\end{tabular}

Deux distinctions importantes apparaissent entre les revues : d'une part, le nombre maximum - et le nombre modal - d'articles par auteur varie. Au maximum, les contributeurs de revues écrivent de 3 à 43 articles; le nombre modal 
est, lui, de 1 à 4. D’autre part, les “classes” d'articles, telles qu'on les a définies, sont elles aussi inégales. La revue RJPEM, par exemple, ne comprend que trois "classes" correspondant chacune à une production d'un article seulement, tandis qu'une revue comme Remald compte cinq "classes" - qui sont, de surcroît, plus importantes, puisque, lors de notre dépouillement, la grande majorité des auteurs avaient publié au moins deux articles. Qui plus est, les contributeurs de la première avaient produit trois articles au maximum, alors que les auteurs les plus prolifiques de la seconde en avaient écrit plus de treize.

Les graphiques ci-dessous illustrent ces écarts : dans le cas de la revue RJPEM, 93,8 \% des articles étaient écrits par de très nombreux auteurs distincts (97,6 \% de l'ensemble des contributeurs de la revue); la production des auteurs les plus prolifiques $(2,4 \%)$ ne représentait, elle, qu'une part minime de tous les articles publiés par la revue (6,2\%). En revanche, 46,5\% seulement des articles de la revue Remald étaient écrits par des auteurs occasionnels (n'ayant publié qu'un, ou deux, ou trois articles), alors que plus du quart des articles étaient dus à quelques contributeurs (2,1\% de l'ensemble).

\section{Graphiques 4 et 5}

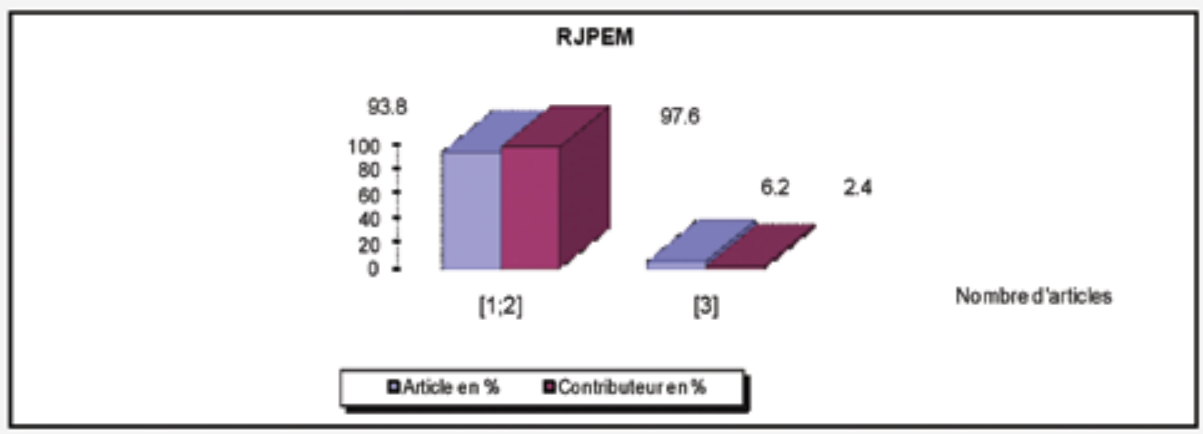

Caractérisation de la production scientifique des revues REMALD et RJPEM

\section{Remald}

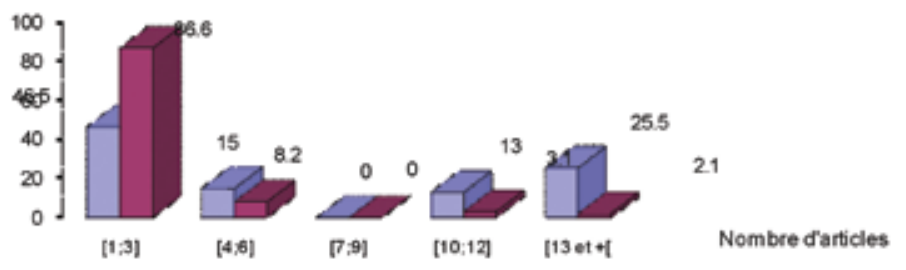


Apparaissent ainsi des “ noyaux” d'auteurs, distingués par la fréquence et le volume importants de leurs contributions à la revue. Suivant nos deux exemples, le noyau de la revue Remald (2,1 \% des contributeurs) est, de façon relative, beaucoup plus "important" que celui de la revue $\operatorname{RJPEM}(2,4 \%)$, puisque, dans le premier cas, les membres de ce noyau participent à $25,5 \%$ de la production totale de la revue - contre 6,2 \% seulement pour le noyau de la seconde revue.

Différents “ cercles " d'auteurs, correspondant aux "classes" précédemment définies de publications par auteur, peuvent également être mis au jour. Certains cercles sont plus proches du noyau des contributeurs réguliers, et plus denses dans la mesure où la production des auteurs, même irrégulière, représente un volume non négligeable d'articles.

D'autres cercles, à l'inverse, sont davantage "périphériques": les chercheurs qui en font partie ne publient dans la revue que de manière occasionnelle (souvent une seule fois).

La structure de la revue Remald peut ainsi être caractérisée par cinq "classes" de productions par auteur, et par autant de "cercles" de contributeurs : de un à trois articles écrits, de quatre à six, de sept à neuf, de dix à douze, enfin de treize articles et plus (le minimum d'articles écrits par auteur étant de un, le maximum étant de quarante-trois). La structure de la revue RJPEM, comme nous l'avons vue, est tout autre.

Le noyau et les cercles de contributeurs varient donc d'une revue à l'autre, selon deux paramètres : le nombre respectif de contributeurs d'une part, le volume d'articles écrits par eux, en proportion de l'ensemble des articles publiés par la revue, d'autre part (voir graphiques pages suivantes ${ }^{2}$ ). Certaines revues ont un " noyau " très restreint d'auteurs qui se détachent par leur contribution plus importante et régulière à la revue ; tel est par exemple le cas de la revue RJPEM. À l'inverse, le noyau de certaines autres revues, de même que les cercles de différents contributeurs, sont l'un et les autres très importants : non seulement les auteurs réguliers et "fidèles" à la revue y publient une proportion importante de toutes les publications, mais la revue compte nombre de contributeurs qui lui proposent des textes en volumes très variables.

C'est le cas, par exemple, des revues Prologues, Annales marocaines d'économie ou encore Remald.

$\overline{2}$ Pour représenter ce noyau, on a choisi de faire correspondre aux contributeurs les plus "productifs" le plus petit cercle. Pour calculer les diamètres des différents cercles, on a donc pris l'inverse du nombre d'articles (plus celui ci est grand, plus le diamètre est petit), multiplié par 10 pour une question d'échelle et de visibilité. Chaque "volume" de contributeurs est repéré par l'intervalle entre deux cercles de même couleur. Le diamètre du second cercle correspond au diamètre du premier, auquel augmenté du nombre de contributeurs divisé par 20. On veut remercier ici P. Giraudbit pour sa collaboration à cette figuration. 
Dans la première (voir graphique), 46,9\% seulement des articles que nous avons dépouillés de 1995 à 2001 avaient été écrits par des auteurs qui n’intervenaient qu'exceptionnellement, à raison de un article ou deux ; 53,1\% des articles avaient donc été écrits par des auteurs qui proposaient trois contributions ou plus (jusqu'à 20 pour l'auteur le plus prolifique). Parallèlement, la revue comptait au moins trois cercles d'auteurs en fonction du volume de leurs contributions. Un premier cercle regroupait des contributeurs qui figuraient de cinq à huit fois dans la revue, un second des auteurs qui apportaient trois à quatre contributions, un dernier enfin de chercheurs qui n'y publiaient qu'une fois ou deux. Comme Prologues, la revue Remald a un noyau de contributeurs plus important que pour les autres revues, et des cercles périphériques d'auteurs qui rédigent une importante proportion d'articles. Ainsi, pour la période que nous avons retenue, $53,5 \%$ des articles étaient écrits par 13,4\% des auteurs, et 2,1\% des auteurs comptabilisaient 25,5\% de l'ensemble des articles écrits sur la période. 8,2\% des auteurs rédigeaient entre quatre et six articles, et 3,1\% de dix à douze.

\section{De La Spécialisation au Monopole de La Publication Scientifique}

Certaines revues accueillent donc très majoritairement des auteurs différents et qui n'interviennent que ponctuellement, voire exceptionnellement. Parallèlement, elles ne reposent que sur très peu d'auteurs “ permanents”, dont la contribution est de surcroît modeste par rapport à l'ensemble des articles publiés.

Réciproquement, certaines revues semblent moins "ouvertes", dans le sens où leur fonctionnement repose pour partie sur un noyau d'auteurs plus important, dont la contribution est parfois majoritaire par rapport à l'ensemble des articles.

Dans ce cas, même si les contributeurs extérieurs au noyau et au(x) premier(s) cercles(s) alimentent fortement la revue, leur place est bien moins importante que pour le premier ensemble de revues, et parfois même secondaire.

Une telle étude du fonctionnement des revues, à travers l'identification de leurs "noyau" et "cercles" de contributeurs d'une part, à travers la répartition des contributions par auteurs d'autre part, apporte ainsi plusieurs enseignements.

En premier lieu, si ce fonctionnement possède des ressorts communs à toutes les revues - en particulier l'existence d'un noyau et de différents cercles ce fonctionnement est assez hétérogène. Schématiquement en effet, certaines revues vivent essentiellement de l'apport ponctuel de contributeurs toujours nouveaux (en particulier les revues RJPEM et Hesperis Tamuda) ; d'autres, à l'inverse (Prologues, Annales marocaines de l'économie ou la revue Remald surtout) bénéficient du concours régulier de cercles restreints de contributeurs et offrent leurs colonnes à des auteurs extérieurs, de manière ponctuelle et exceptionnelle, 
dans une bien moindre mesure.

Faute d'investigations qualitatives (auprès des comités de rédaction de revue notamment), on se gardera ici d'aller trop loin dans l'interprétation d'une telle donnée. Ignorant si cette structuration ressortit à une demande (forte ou faible) de contributeurs extérieurs à chaque revue, ou à une politique d'édition particulière de l'une à l'autre, on ne saurait en effet apporter de conclusions fermes sur ces apparentes "fermeture" ou "ouverture" — au demeurant relatives — des différentes revues. On avancera l'hypothèse (pour s'en assurer il faudrait lire l'ensemble des articles ici répertoriés) que cette "fermeture" et cette "ouverture" constituent des indices de structuration du champ de production et de diffusion des recherches. Certaines revues, en effet, semblent privilégier les résultats de recherche propres au champ scientifique marocain, tandis que d'autres paraissent s'ouvrir davantage aux recherches externes à ce champ.

On notera aussi que chaque discipline a visiblement des modes différents, pluriels, de production et de diffusion des résultats de recherche. Pour l'économie, par exemple, les revues RJPEM et les Annales marocaines d'économie sont caractéristiques puisqu'elles présentent très nettement les deux schémas de fonctionnement précédemment distingués. On notera aussi, plus justement peut-être, qu'il n'y a pas — et ce, pour aucune discipline - de mode unique de production et de diffusion des recherches. Chaque "champ disciplinaire" est en lui-même hétérogène : il recourt à des modes différenciés de production, de publication et de diffusion des recherches, que celles-ci lui soient propres (internes) ou extérieures.

Une investigation plus poussée permet cependant d'être plus précis : manifestement, pour les revues marocaines étudiées, c'est la longévité de la revue - son histoire, donc - qui module son fonctionnement. La participation de chercheurs extérieurs au noyau est en effet toujours plus nette pour les jeunes revues que pour celles qui ont dépassé un nombre important de numéros. Conjointement, le noyau des revues anciennes est plus important (en volume de publications), et la contribution des auteurs occasionnels proportionnellement moindre. Un support comme la revue scientifique semble ainsi permettre la réunion de chercheurs et semble fonctionner petit à petit comme un pôle de production scientifique, de reconnaissance (scientifique, s'entend) de chercheurs - par l'audience que représentent la publication et sa diffusion - et, tout autant, comme un espace de visibilité institutionnelle des recherches. Cette visibilité et cette reconnaissance des chercheurs et des recherches configurent le champ de la recherche lui-même. En effet, non seulement elles signalent la "réussite" de chercheurs ou de groupes de chercheurs dans le procès de reconnaissance auquel ils sont soumis de la part de leurs pairs (Bourdieu, 1975, 1991; Latour \& Woolgar, 1979, (Latour, 1995), mais elles fonctionnent comme un aimant pour 
Graphique 6 - Répartition des contributeurs selon le nombre d'articles - Revue Juridique, Politique et Economique du Maroc

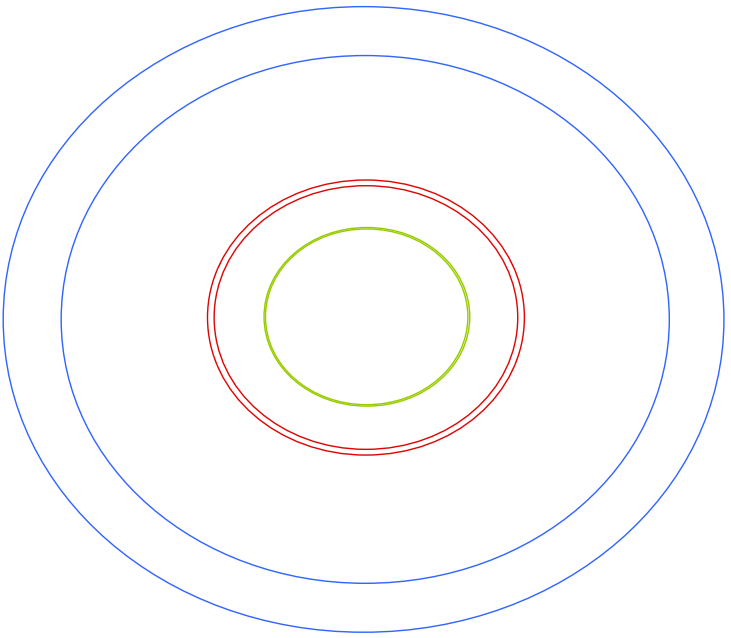

Légend e : Nombr e d'articles

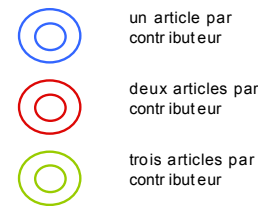

Nombr e de cont ributeurs

Graphique 7 - Répartition des contributeurs selon le nombre d'articles Revue Prologues

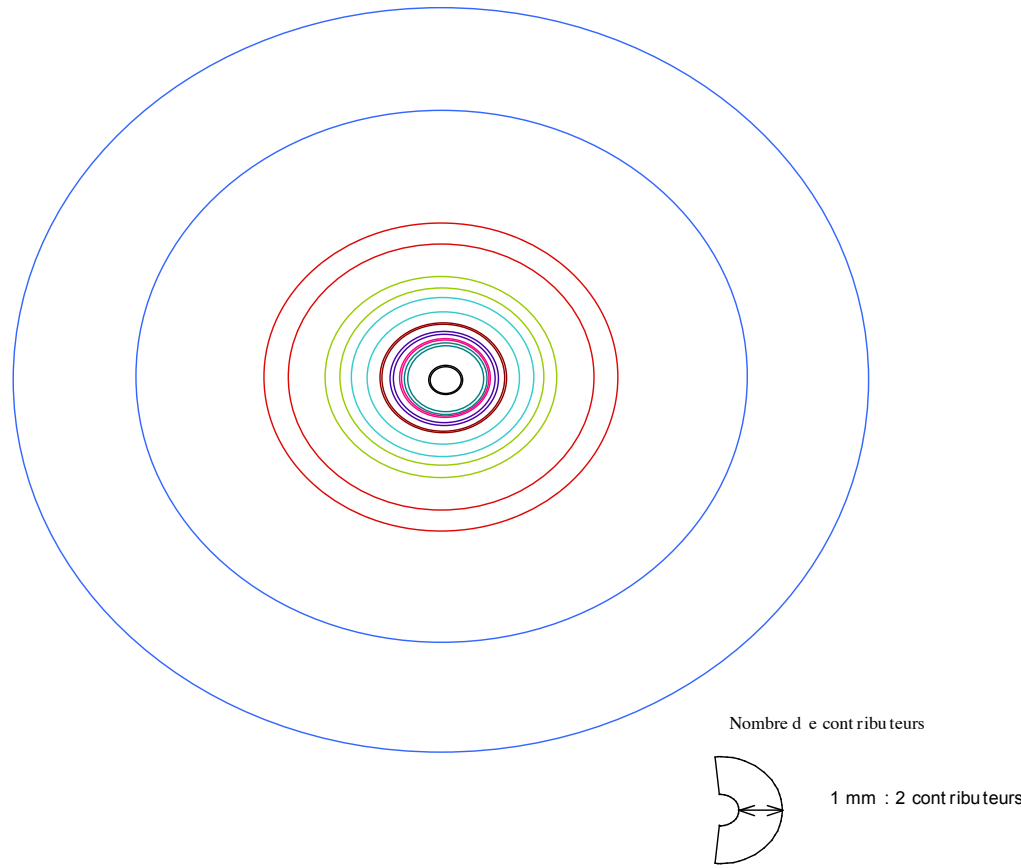

Légend e : Nombr e d'a

(O) un art

(O) $\begin{aligned} & \text { deux a } \\ & \text { contrit }\end{aligned}$

(2) trois a

(1) quatre

(O) cinq a

(D) six art

(O) sept al

(D) huit a

(O) ${ }_{\text {par col }}^{\text {vingt }}$ 
tout chercheur extérieur ( il faut absolument que tu publies là ») et contribuent, en retour, à l'émergence de ce que l'on a pu désigner par “école” (i.e. de pensée ${ }^{3}$ ).

Comme le signale B. Godin, "les chercheurs, dont le capital scientifique est tributaire de l'audience potentielle, tendent évidemment à maximiser l'impact de leurs travaux en essayant de publier dans les revues les plus largement diffusées " (Godin, 2002 : 466), en l'occurrence celles où publient les chercheurs qui ont obtenu une consécration de leurs pairs.

La comparaison des revues marocaines dépouillées (de la structure de leur production en noyau et cercles périphériques) suggère donc qu'à mesure de son évolution, la majorité d'entre elles se resserrent autour d'un noyau de contributeurs principaux et enregistrent une participation de contributeurs extérieurs plus faible qu'elle ne l'était à la naissance et dans les premiers temps de la revue.

Chaque revue semble peu à peu se "recentrer" sur elle-même — soit qu'elle ne parvient pas à fidéliser ses contributeurs, soit que sa diffusion ne lui permette pas un apport régulier de contributeurs occasionnels. À l'étude, on voit ainsi que le fonctionnement de la revue scientifique de SHS au Maroc ne va généralement pas dans le sens d'une "externalité" mais, au contraire, dans celui d'un recentrage sur elle-même.

Cette donnée n'est pas générale, loin s'en faut. L'analyse d'une autre revue scientifique, française cette fois - analyse que l'on ne détaillera pas ici - a pu au contraire nous montrer que la longévité de la revue allait de pair avec la contribution, toujours plus importante quantitativement, de chercheurs extérieurs au cercle des fondateurs.

$\mathrm{Si}$ cette revue spécialisée pouvait également constituer un pôle quant aux publications sur son objet principal (l'éducation), elle ne le devait qu'à cette agrégation constante de chercheurs extérieurs, proportionnellement de plus en plus importants par rapport au noyau initial de la revue.

Cette étude sur le fonctionnement de la production scientifique des SHS au Maroc invite ainsi à émettre l'hypothèse que le mode de recherche — voire le fonctionnement des équipes de chercheurs, et des chercheurs eux-mêmes répond à une logique particulière, allant dans le sens d'une concentration importante.

À mesure de son évolution, ce champ de la recherche semble ainsi atomisé en un ensemble de "groupes" de chercheurs communs par leur discipline et, à la fois, "concentré" en quelques "groupes" qui produisent prioritairement autour d'eux-mêmes.

3 En France, une revue vient spontanément à l'esprit à cet égard : les Actes de la recherche en sciences sociales, très longtemps dirigée par Pierre Bourdieu. 
Cette caractéristique, qui demanderait des recherches qualitatives complémentaires, indique l'une des directions possibles pour une éventuelle recherche sur les SHS : une étude du fonctionnement du champ global de ces sciences peut prendre pour base l'identification des noyaux de chercheurs (et non plus de contributeurs - avec lesquels il faudrait néanmoins les comparer), par discipline et appartenance institutionnelle, et se prolonger par l'étude des modes de collaboration/coopération de ces chercheurs, entre eux et avec d'autres, plus occasionnels.

Enfin, s'il s'agit d'identifier le mode de travail et de coopération possible, avec les chercheurs en SHS, il apparaît nécessaire d'identifier les principes et règles à la base de cette logique de fonctionnement : quelles peuvent être, notamment, les contraintes à la source de cette structuration en noyaux et de ce recentrage des chercheurs sur eux-mêmes?

\section{Conclusion}

Nous avons pu montrer dans un autre texte que certains objets scientifiques sont davantage abordés et "traités" par certaines sciences sociales, de sorte que le travail et la production scientifiques respectent une certaine "focalisation disciplinaire »(Gérard \& Kleiche, 2006).

L'une des conclusions apportées par ce volet d'étude sur les revues converge avec cette dernière donnée.

En effet, la structure des revues et leur mode de publication se modifient avec le temps : au départ largement alimentées par un grand nombre de contributeurs, dont les apports sont ponctuels, les revues se recentrent autour d'un nombre restreint de contributeurs, dont l'apport prend peu à peu de l'importance par rapport à celui des "cercles" périphériques d'auteurs extérieurs et occasionnels.

À une focalisation disciplinaire correspond ainsi un certain recentrage des revues sur elles-mêmes - donc un certain regroupement des chercheurs autour d'objets scientifiques, de normes de publication, ou encore de modèles de traitement de ces objets.

L'assertion corollaire semble vraie également : alors que certains thèmes font l'objet, à travers la focalisation des chercheurs, d'un approfondissement qui prend pour traits ceux d'une spécialisation — voire d'une spécificité — disciplinaire, des supports de publication comme les revues se resserrent aussi autour de certains objets scientifiques, et doivent probablement l'importance croissante de leur noyau de contributeurs à cette spécialisation et à la visibilité peu à peu acquise par la revue ; en somme, à un certain monopole de la production scientifique. 


\section{Bibliographie}

Bourdieu (P.), 1975, La spécificité du champ scientifique et les conditions du progrès de la raison Sociologie et société, VII, 1, pp. 91-119.

Bourdieu (P.), 1971, Le marché des biens symboliques, L'année sociologique, 22, pp. 49-126.

Gérard (E.) et Kleiche-Dray (M.), 2006, La publication comme indicateur de la structuration du champ scientifique : analyse à partir des sciences humaines et sociales au Maroc, Prologues, Casablanca, n³6, pp. 66-79.

Godin (B.), 2002, Les pratiques de publication des chercheurs : les revues savantes québécoises entre impact national et visibilité internationale, Recherches sociographiques, XLIII, pp. 465-498.

Latour (B.) \& Woolgart (S.), La vie de laboratoire : la production des faits scientifiques, Paris : La Découverte.

Latour (B.), 1995, La science en action, Paris: Gallimard. 